\title{
ELEMENTARY OPERATIONS WHICH GENERATE NETWORK MATRICES'
}

\section{R. J. DUFFIN}

In this note some of the interesting work on the synthesis problem of electrical network theory is translated into ordinary algebraic language.

A function $f(z)$ of a complex variable $z$ is positive real in the sense of Brune if $f(z)$ is a rational function with real coefficients such that $\operatorname{Re} f(z) \geqq 0$ for $\operatorname{Re} z \geqq 0$. (PR is an abbreviation for positive real.) Starting with PR functions it is obvious that the following operations lead to new PR functions:

(a) Multiplication by a positive constant or zero, $c f(z)$;

(b) Forming the inverse, $1 / f(z)$;

(c) Addition, $f_{1}(z)+f_{2}(z)$.

The zero function is PR and operation (b) is excluded in this case.

Starting with the PR functions 1 and $z$ as a basis, it follows that the operations (a), (b), and (c) generate a subclass of the PR class. It is a consequence of a synthesis method given by Bott and Duffin [1] that actually any PR function can be so generated. This observation is due to Brockway McMillan. In this note it is shown that the class of positive real matrices may be generated in an analogous fashion.

A positive real matrix function $F(z)$ is defined as follows:

I. $F$ is an $n$ by $n$ symmetric matrix.

II. The matrix elements $F_{j k}$ are rational functions of $z$ with real coefficients.

III. For any choice of complex numbers $c_{1}, c_{2}, \cdots, c_{n}$

$$
\operatorname{Re} \sum_{1}^{n} \sum_{1}^{n} F_{j k} c_{k} c_{j}^{*} \geqq 0 \quad \text { for } \quad \operatorname{Re} z \geqq 0 .
$$

Here the asterisk denotes the complex conjugate. Starting with PR matrices the following elementary operations lead to new PR matrices:

(a) $A_{t} F A$ where $A$ is an arbitrary $n$ by $n$ matrix with real constant matrix elements and where $A_{t}$ is the transpose of $A$;

(b) Forming the inverse, $F^{-1}$ (if its exists);

Received by the editors July 6, 1954.

1 The work on this paper was sponsored by the Office of Ordnance Research, U.S. Army, Contract DA-36-061-ORD-378. 
(c) Addition, $F_{1}+F_{2}$.

It is obvious that (a) and (c) lead to PR matrices. It is clear that $F^{-1}$ satisfies I and II. Let $\sum F_{j k}^{-1} c_{k}=b_{j}$; then

$$
\sum \sum F_{j k}^{-1} c_{k} c_{j}^{*}=\sum b_{j} c_{j}^{*}=\left(\sum \sum F_{j k} b_{k} b_{j}^{*}\right)^{*}
$$

and it is seen that III is also satisfied. Thus $F^{-1}$ is PR.

The following theorem is to be proved:

THEOREM. Starting with the identity matrix $I$ and the matrix $z I$ as a basis, then any positive real matrix function $F(z)$ may be generated by the operations (a), (b), and (c) in a finite number of steps.

The proof is to be given by induction on the number of rows and columns. In the case $n=1$, a PR matrix is a PR function and the operations (a), (b), and (c) for matrix functions are identical with the operations (a), (b), and (c) for functions. It is easily seen that the cited paper of Bott and Duffin furnishes a proof in this case. The procedure given there is based on a version of Schwarz's lemma discovered by P. I. Richards.

The procedure which follows is based on a series of theorems stated by M. Bayard [2]. The proofs of Bayard's theorems are given in a paper by R. Leroy [4]. The work of Bayard and Leroy is based on previous work of C. M. Gewertz and W. Cauer. To suit the present purpose these theorems will be stated here in a slightly modified form. Some of the proofs are outlined; for more detail the paper of Leroy as well as the other references cited may be consulted.

Suppose that the theorem is true for $n-1$ by $n-1$ matrices and consider an $n$ by $n$ PR matrix $F$. First suppose that

$$
\operatorname{det} F(z) \equiv 0
$$

for all $z$.

For $z=1, F$ is a real symmetric matrix. By a standard diagonalization theorem there exists a matrix $E$ which is real, constant, and nonsingular such that $G=E_{t} F E$ is diagonal at the point $z=1$. Because of (1) some diagonal element of $G(1)$ must vanish. Without loss of generality it may be assumed that $G_{n n}(1)=0$. It follows from III that the diagonal elements of a PR matrix are PR functions. It is easy to see that 0 is the only PR function which can vanish for $\operatorname{Re} z>0$. Thus $G_{n n}(z) \equiv 0$. It then follows from III that $G_{n j}(z) \equiv 0$ for $j=1, \cdots, n$.

Let $G^{\prime}$ be an $n-1$ by $n-1$ matrix whose matrix elements are $G_{j k}$ for $j, k=1,2, \cdots, n-1$. Let $I^{\prime}$ denote the $n-1$ by $n-1$ identity. Then by the inductive hypothesis $G^{\prime}$ can be generated from $I^{\prime}$ and $z I^{\prime}$. In the course of this generation constant multiplier matrices $A^{\prime}$ 
may be employed. With each such matrix associate an $n$ by $n$ matrix $A$ defined as $A_{j k}=A_{j k}^{\prime}$ for $j, k=1,2, \cdots, n-1$ and $A_{n n}=1$ and $A_{j n}=A_{n j}=0$ if $j \neq n$. Then the operations which generate $G^{\prime}$ from $I^{\prime}$ and $z I^{\prime}$ are applied to $I$ and $z I$ with $A$ replacing $A^{\prime}$. If $G^{\prime \prime}$ is the matrix so generated, it is clear that $G_{k j}^{\prime \prime}=G_{k j}^{\prime}=G_{k j}$ for $k, j=1,2, \cdots, n-1$. Let $D=I$ except that $D_{n n}=0$, so $G=D_{t} G^{\prime \prime} D$ and

$$
F=E_{t}^{-1} D_{t} G^{\prime \prime} D E^{-1} \text {. }
$$

Thus $F$ is generated from $G^{\prime \prime}$ by operation (a) with $A=D E^{-1}$.

Let $c_{1}, c_{2}, \cdots, c_{n}$ be an arbitrary set of complex constants, then

$$
f(z)=\sum_{1}^{n} \sum_{1}^{n} F_{j k}(z) c_{k} c_{j}^{*}
$$

is a PR function if $F$ is a PR matrix. It is seen from (3) that $F$ can have no poles for $\operatorname{Re} z>0$. Moreover, poles on the imaginary axis must be simple. As a convention poles at infinity are here regarded as being "on the imaginary axis."

A PR matrix $\Delta$ which is an odd function of $z$ is termed IPR. Clearly the only poles of $\Delta$ are simple poles on the imaginary axis. Thus $\Delta$ may be expanded in the partial fraction form

(4) $\Delta(z)=z B_{\infty}+z^{-1} B_{0}+z\left(z^{2}+k_{1}^{2}\right)^{-1} B_{1}+\cdots+z\left(z^{2}+k_{m}^{2}\right) B_{m}$

where $k_{1}, \cdots, k_{m}$ are real distinct positive constants and where the matrices $B_{\infty}, B_{0}, B_{1}, \cdots, B_{m}$ are real, constant, and symmetric. From (3) it is seen that the $B$ matrices are semi-definite. Any semidefinite matrix $B$ can be expressed in the form $B=A_{t} A$ where $A$ is a real matrix. Thus

$$
z\left(z^{2}+k^{2}\right)^{-1} B=A_{t}\left[z I+k^{2}(z I)^{-1}\right]^{-1} A .
$$

It follows from (4) and (5) that any IPR matrix $\Delta$ can be generated. A PR matrix $Q$ whose matrix elements are bounded on the imaginary axis may be termed BPR. By expansion in partial fractions any $\mathrm{PR}$ matrix $F$ may be decomposed in the form

$$
F=\Delta+Q
$$

where $\Delta$ is IPR and $Q$ is BPR.

If $Q$ is a BPR matrix let a matrix $Q^{0}$ be defined by

$$
2 Q^{0}(z)=Q(z)+Q(-z) .
$$

Thus $Q^{0}(z)$ is an even function of $z$. On the imaginary axis, $z=i y$, clearly 


$$
Q^{0}(i y)=\operatorname{Re} Q(i y) .
$$

Thus $Q^{0}(i y)$ is a real semi-definite matrix and is a function of $y^{2}$. By the Gauss method of completing the square, a semi-definite quadratic form may be expressed as a sum of $m$ squares of linear forms $(m \leqq n)$. Associating a matrix with each square gives

$$
Q^{0}(z)=\sum_{1}^{m} R_{j}^{0}(z)
$$

Here each matrix $R_{f}^{0}(z)$ is symmetric and the matrix elements are rational functions of $z^{2}$ with real coefficients. $R_{j}^{0}(i y)$ is semi-definite, so it follows from (9) that $R_{j}^{0}(i y)$ can not have a pole on the imaginary axis, for othersise $Q^{0}(i y)$ would have a pole. The quadratic form of $R_{j}^{0}(z)$ is the square of a linear form, so the matrix $R_{j}^{0}(z)$ has rank 1 with the possible exception of a finite number of $z$ values. Except for this last property $R_{f}^{0}(z)$ has the same properties as $Q^{0}(z)$.

By a partial fraction expansion of $R_{f}^{0}(z)$, it is seen that there is a matrix $R_{j}(z)$ without poles for $\operatorname{Re} z \geqq 0$ such that

$$
2 R_{j}^{0}(z)=R_{j}(z)+R_{j}(-z) .
$$

Let $g(z)$ be a function which is real on the real axis and which is analytic for $\operatorname{Re} z \geqq 0$ including the point at infinity. Then $g(z)$ is uniquely determined by the value of its real part on the imaginary axis. Thus

$$
Q(z)=\sum_{1}^{m} R_{j}(z)
$$

Moreover, by the same lemma it is seen that $R_{j}(z)$ is BPR. The decomposition relation (11) was found independently by Y. Oono [5].

By virtue of (11) the problem has been reduced to the consideration of a BPR matrix $R$ whose even part $R^{0}$ is of rank 1 ; these are termed regular reduced matrices by Leroy. The case $\operatorname{det} R \equiv 0$ has been disposed of, so it may be assumed that $R$ has an inverse, say $X$. Then

$$
R^{-1}=X=\nabla+P
$$

where $\nabla$ is IPR and $P$ is BPR. Since $X(z)+X(-z)=X(z)[R(z)$ $+R(-z)] X(-z)$, it is seen that

$$
P^{0}(z)=X^{0}(z)=X(z) R^{0}(z) X(-z) .
$$

It follows that the even part of $P$ is also of rank 1 .

Leroy considers the degree of a matrix of rational functions to be 
the degree of the lowest common denominator polynomial of the matrix elements. He shows that the degree of $R$ is greater than the degree of $P$. Thus if the theorem is not true, there is a BPR matrix $R$ whose even part is of rank 1 and of lowest degree which can not be generated. But (12) shows that $R$ can be generated from $\nabla$ and $P$, and this contradiction completes the proof of the theorem.

A different definition of degree was introduced by B. McMillan [6] and independently by $\mathrm{B}$. Tellegen [7]. The degree of $F$ may be defined as the maximum of the degree of the numerator polynomial of $\operatorname{det}(F+A)$ for any constant matrix $A$. It results from McMillan's theorems that degree $R$ =degree $R^{-1}=$ degree $\nabla+$ degree $P$. For $z=0$, det $R=\operatorname{det} R^{0}=0$. Thus det $R^{-1}$ has a pole at $z=0$, and so some of the matrix elements of $\nabla$ have a pole at $z=0$. It follows that degree $R>$ degree $P$, and so an alternative proof of the theorem is furnished.

The references cited below give various methods of synthesizing a network with $n$ pairs of terminals. The theorem proved here furnishes another solution of this synthesis problem. Operation (a) corresponds to the use of an ideal transformer; operation (b) corresponds to changing from impedance to admittance; and operation (c) corresponds to series or parallel connection. The synthetic network resulting may be regarded as a generalized series-parallel network.

\section{REFERENCES}

1. R. Bott and R. J. Duffin, Impedance synthesis without use of transformers, Journal of Applied Physics vol. 20 (1949) p. 816.

2. M. Bayard, Synthèse des reseaux passifs d un nombre quelconque de paires de bornes connaisant leurs matrices d'impédances ou d'admittances, Bulletin Société Francaise Electriciens vol. 9 (1949) pp. 497-502.

3. - Synthesis of n-terminal pair networks, Proc. Symposium on Modern Network Synthesis, Polytechnic Institute of Brooklyn, 1952.

4. R. Leroy, Sur la synthèse des reseaux passifs $d n$ paires de bornes, Cables and Transmission vol. 4 (1950) pp. 234-247.

5. Y. Oono, Synthesis of a finite 2 -terminal network by a group of networks each of which contains only one ohmic resistance, Journal of Mathematics and Physics vol. 29 (1950) pp. 13-26.

6. B. McMillan, Introduction to formal realizability theory, The Bell System Technical Journal vol. 31 (1952) pp. 217-299 and pp. 541-600.

7. B. D. H. Tellegen, Synthesis of $2 n$-poles by networks containing the minimum number of elements, Journal of Mathematics and Physics vol. 32 (1953) pp. 1-18.

Carnegie Institute of Technology 